\title{
Transfer Function Method for the Nonlinear Flutter of a High-aspect-ratio Wing
}

\author{
Zhongyuan Zhang ${ }^{1}$, Jingbo Duan ${ }^{2}$ and Ping $\mathrm{Lu}^{2}$ \\ ${ }^{1}$ Department of UAV Engineering, Army Engineering University, Shijiazhuang, China \\ ${ }^{2}$ Mechanics Engineering Department Shijiazhuang Tiedao University, Shijiazhuang, China
}

\begin{abstract}
The semi-analytical and semi-numerical transfer function method is applied to the nonlinear flutter of a high-aspect-ratio aircraft wing. Firstly, the flutter differential equation of a three-dimensional wing is established by combining the bend-twist vibration equations of the wing and the ONERA nonlinear aerodynamics model for wings. Then, using the transfer function method, the control equations are formulated in a state-space form by defining a state vector. Both the flutter velocity and flutter frequency are obtained by solving a complex eigenvalue problem. Finally, the differences of the flutter behavior of the linear and nonlinear aerodynamic models under the influence of parameters such as mass of unit length, semi-aspect ratio, the stiffness ratio of twist and bend are discussed.
\end{abstract}

Keywords-high-aspect-ratio wing; nonlinear aerodynamic force; flutter; transfer function method

\section{INTRODUCTION}

Flutter has always been an important issue in aircraft design. Once flutter occurs, the consequences are catastrophic, killing planes in seconds. For example, an F-117 stealth fighter crashed in 1997 due to maneuvering flutter[1]. Thus, flutter analysis plays an important role in the design of the aircraft.

Wing is an important part of aircraft for aerodynamic analysis. Domestic and foreign scholars conducted an in-depth study of flutter. In the 1990s, MJ Patil[2] et al. Proposed the method of limit cycle and its theoretical analysis and experimental research. Zhouzhou team[3,4] for this issue, conducted in-depth study and made considerable progress, large flexible high aspect ratio solar UAV analysis has reference value; $\mathrm{R}$ Palacios[5] used three-dimensional Euler equations modeling to achieve a detailed three-dimensional representation of aerodynamics and structural mechanics. Z Sotoudeh[6] developed aeroelastic analysis of a flexible UAV at high altitude, A set of computer programs specially designed for such UAVs can be used to obtain the results of aeroelastic analysis in a short period of time, which is convenient for the design of flexible UAVs. D Tang[7] combined the aeroelastic analysis of flexible wings with the wind Hole test, a theoretical aeroelastic model of aeroelastic model with flexible large aspect ratio airfoil under elastic load is introduced. MJ Patil et al.[8,9] proposed the aeroelastic behavior of a complete aircraft model and the results obtained from the analysis of the overall flight dynamics. Due to the flexibility of the wing, the overall flight dynamics of the aircraft also changed, Linear aeroelastic analysis to explain this behavior.
In this paper, ONERA nonlinear aerodynamic model is used to deal with the problem of high aspect ratio wing flutter based on the transfer function method. The transfer function method is a semi-analytical semi-numerical method based on control theory. The method has simple and uniform solution process, and the boundary conditions are normalized and convenient. The method is often used to analyze the stability and dynamic response of dynamic system.

\section{FLUTTER DifFERENTIAL EQUATION OF THE WING}

The flutter analysis of high aspect ratio rigid straight wing, the solution of this paper is as follows: Firstly, the flutter differential equation is obtained according to the differential equations of bending and torsional vibration of the clean wing and the nonlinear ONERA aerodynamic model, vibration differential equations is prepared for the transfer function method.

\section{A. The Bend-twist Vibration Equations}

The object of study is a long, straight clean wing with a half-span $L$ and a half-chord $b$. The differential equations of the bending and torsional vibration of the clean wing can be written as

$$
\left\{\begin{array}{l}
E I \frac{\partial^{4} h}{\partial y^{4}}+m \frac{\partial^{2} h}{\partial t^{2}}-m x_{\alpha} \frac{\partial^{2} \alpha}{\partial t^{2}}-L_{h}=0 \\
G J \frac{\partial^{2} \alpha}{\partial y^{2}}-I_{\alpha} \frac{\partial^{2} \alpha}{\partial t^{2}}+m x_{\alpha} \frac{\partial^{2} h}{\partial t^{2}}+T_{\alpha}=0
\end{array}\right.
$$

where $h$ is the wing bending vibration displacement, $\alpha$ is the wing torsional vibration angle, $E I$ is the wing flexural rigidity, $G J$ is the wing torsional stiffness, $m$ is the mass of unit length, $I_{\alpha}$ is the unit moment of inertia of the wing around the elastic axis, $X_{\alpha}$ is the distance between the elastic axis of the wing and the center of gravity of the wing section, $L_{h}$ is the lift per unit length of the wing, $T_{\alpha}$ is the torque per unit length of the wing, $y$ is the span direction of the wing and $t$ is the time. 


\section{B. ONERA Aerodynamic Model}

Ignoring the effects of wing gravity, the external forces on the wing flutter are only aerodynamic. The ONERA dynamic model is used here to describe the nonlinear unsteady aerodynamics. In different literatures, the expression forms are also different. The ONERA dynamic model introduced in literature [10] is used and the expression is:

$$
\left\{\begin{array}{l}
C_{z}=C_{z a}+C_{z b} \\
C_{z a}=\frac{b}{V} S_{z 1} \frac{\partial \alpha}{\partial t}+\left(\frac{b}{V}\right)^{2} S_{z 2} \frac{\partial^{2} \theta}{\partial t^{2}}+\frac{b}{V} S_{z 3} \frac{\partial \theta}{\partial t}+C_{z \gamma} \\
\frac{b}{V} \frac{\partial C_{z \gamma}}{\partial t}+\lambda_{1} C_{z \gamma}=\lambda_{1} a_{o z}\left(\alpha+\frac{b}{V} \frac{\partial \theta}{\partial t}\right)+\lambda_{2} a_{o z}\left(\frac{b}{V} \frac{\partial \alpha}{\partial t}+\left(\frac{b}{V}\right)^{2} \frac{\partial^{2} \theta}{\partial t^{2}}\right) \\
\left(\frac{b}{V}\right)^{2} \frac{\partial^{2} C_{z b}}{\partial t^{2}}+\frac{b}{V} r_{1 z} \frac{\partial C_{z b}}{\partial t}+r_{2 z} C_{z b}=-r_{2 z} \Delta C_{z}-\frac{b}{V} r_{3 z} \frac{\partial \Delta C_{z}}{\partial \alpha} \frac{\partial \alpha}{\partial t}
\end{array}\right.
$$

Here, $V$ is the velocity of the incoming stream, $\theta$ is the instantaneous angle of attack, $b$ is the half-chord of the airfoil and $C_{z}$ represents the aerodynamic coefficient, $C_{z a}$ represents the aerodynamic function corresponding to the linear aerodynamic part and $C_{z b}$ represents the aerodynamic coefficient corresponding to the nonlinear aerodynamic part. Parameters subscript is $Z=L$, said lift-related coefficients; when the subscript is $Z=M$, said the torque-related coefficients.

TABLE I. THE PARAMETERS OF THE ONERA

\begin{tabular}{|c|c|c|}
\hline coefficients & lift & torque \\
\hline$S_{z 1}$ & $S_{L 1}=\pi$ & $s_{M 1}=-\pi / 4$ \\
\hline$S_{z 2}$ & $S_{L 2}=\pi / 2$ & $s_{M 2}=-3 \pi / 16$ \\
\hline$s_{z 3}$ & $S_{L 3}=0.0$ & $s_{M 3}=-\pi / 4$ \\
\hline$a_{o z}$ & $a_{o L}=5.9$ & $a_{o M}=0.0$ \\
\hline$\lambda_{1}$ & & 0.15 \\
\hline$\lambda_{2}$ & \multicolumn{2}{|c|}{0.55} \\
\hline
\end{tabular}

To simplify the calculation, it is common to use a polyline instead of a static aerodynamic curve, as shown in Figure 1.

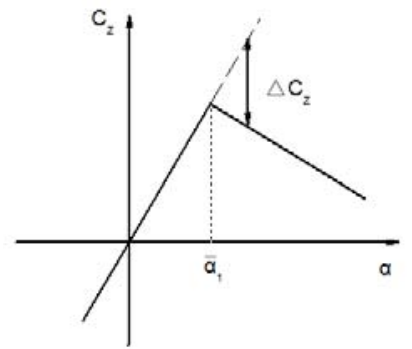

FIGURE I. STATIC AERODYNAMIC APPROXIMATE FIGURE

The relationship between the instantaneous angle of attack $\theta$ and the effective angle of attack $\alpha$ in equation (2) is:

$$
\alpha=\theta-\frac{\dot{h}_{1 / 4}}{V}
$$

where, $h_{1 / 4}=\frac{h-(0.5 b+\bar{a} b) \alpha}{b}$, Substitution equation

and finishing:

$$
\theta=\alpha+\frac{\frac{\partial h}{\partial t}-(0.5 b+\bar{a} b) \frac{\partial \alpha}{\partial t}}{b V}
$$

The unit lift and the corresponding pitch moment can be expressed as:

$$
\left\{\begin{array}{l}
L_{h}=b \rho V^{2} C_{L} \\
T_{\alpha}=2 \rho b^{2} V^{2} C_{M}
\end{array}\right.
$$

where, $C_{L}$ and $C_{M}$ are obtained by the equation (2), Substitute (5) into equation (1), you can get a high aspect ratio wing flutter differential equations.

$$
\left\{\begin{array}{l}
E I \frac{\partial^{4} h}{\partial y^{4}}+m \frac{\partial^{2} h}{\partial t^{2}}-m x_{\alpha} \frac{\partial^{2} \alpha}{\partial t^{2}}-b \rho V^{2} C_{L}=0 \\
G J \frac{\partial^{2} \alpha}{\partial y^{2}}-I_{\alpha} \frac{\partial^{2} \alpha}{\partial t^{2}}+m x_{\alpha} \frac{\partial^{2} h}{\partial t^{2}}+2 \rho b^{2} V^{2} C_{M}=0
\end{array}\right.
$$

\section{TRANSFER FUNCTION METHOD}

Using fourier transform on equation (6), one can obtain:

$$
\left\{\begin{array}{l}
\frac{\partial^{4} h}{\partial y^{4}}=A_{1}(\omega, V) h+B_{1}(\omega, V) \alpha \\
\frac{\partial^{2} \alpha}{\partial y^{2}}=A_{2}(\omega, V) h+B_{2}(\omega, V) \alpha
\end{array}\right.
$$


The state vector can be defined as:

$$
\boldsymbol{\eta}_{e}(y, \omega)=\left[\begin{array}{llllll}
h & \frac{\partial h}{\partial y} & \frac{\partial^{2} h}{\partial y^{2}} & \frac{\partial^{3} h}{\partial y^{3}} & \alpha & \frac{\partial \alpha}{\partial y}
\end{array}\right]^{\mathrm{T}}
$$

Then, equation (7) can be written in state space form:

$$
\frac{\partial \boldsymbol{\eta}(y, \omega)}{\partial y}=\mathbf{F}(\omega, V) \boldsymbol{\eta}(y, \omega)+\mathbf{g}(y, \omega)
$$

Where, $\mathbf{g}(y, \omega)=\mathbf{0}$.

The boundary condition is:

$$
\mathbf{M}_{b} \boldsymbol{\eta}_{e}(0, \omega)+\mathbf{N}_{b} \boldsymbol{\eta}_{e}(1, \omega)=\boldsymbol{\gamma}_{e}(\omega)
$$

Where, $\mathbf{M}_{b}$ is selected matrix for the wing root boundary conditions, and $\mathbf{N}_{b}$ is selected matrix for the wing tip boundary conditions.

According to the transfer function theory [11], the solution of (9) can be written as:

$$
\boldsymbol{\eta}_{e}(y, \omega)=\mathbf{H}_{e}(y, \omega, V) \boldsymbol{\gamma}_{e}(\omega)+\mathbf{f}_{e}(y, \omega)
$$

where,

$$
\begin{aligned}
& \mathbf{H}_{e}(y, \omega, V)=\boldsymbol{\Phi}_{F}(0, \omega, V)\left[\mathbf{M}_{b} \mathbf{\Phi}_{F}(0, \omega, V)+\mathbf{N}_{b} \boldsymbol{\Phi}_{F}(0, \omega, V)\right]^{-1}, \\
& \boldsymbol{\Phi}_{F}(y, \zeta, \omega, V)=e^{\mathbf{F}(y, \omega, V) \zeta}
\end{aligned}
$$

For the entire wing, the general solution equation can draw on the idea of finite element method for grouping.

\section{Flutter Analysis For the High AsPect Ratio Wing}

A wing model is selected for flutter calculation of nonlinear aerodynamic force and linear aerodynamic force. The wing model is shown in Table 2, the flutter results with linear and nonlinear aerodynamic models were compared by changing the parameters such as mass of nuit length, aspect ratios, stiffness of bend and stiffness of twist.

TABLE II. THE PARAMETERS OF THE AIRCRAFT WING

\begin{tabular}{|c|c|}
\hline Half of span(m) & 16 \\
\hline Half of chord(m) & 1 \\
\hline Mass of nuit length $(\mathrm{kg} / \mathrm{m})$ & 79 \\
\hline $\begin{array}{c}\text { Position of the elastic } \\
\text { axis(\%)hord) }\end{array}$ & 50 \\
\hline Stiffness of bend $\left(\mathrm{N} \cdot \mathrm{m}^{2}\right)$ & $2 \times 10^{8}$ \\
\hline Stiffness of twist $\left(\mathrm{N} \cdot \mathrm{m}^{2}\right)$ & $3 \times 10^{8}$ \\
\hline
\end{tabular}

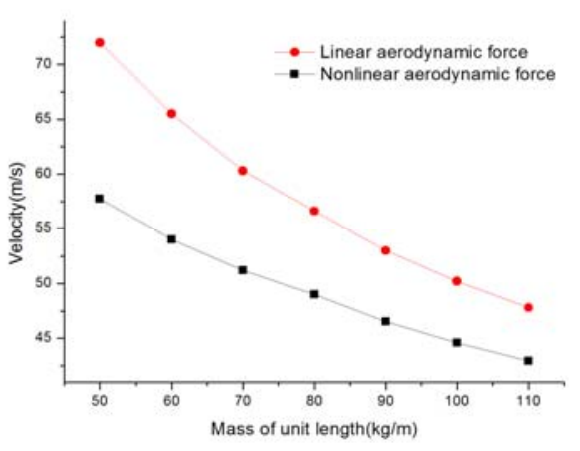

FIGURE II. THE INFLUENCE OF THE MASS OF UNIT LENGTH ON THE VELOCITY OF THE WING FLUTTER

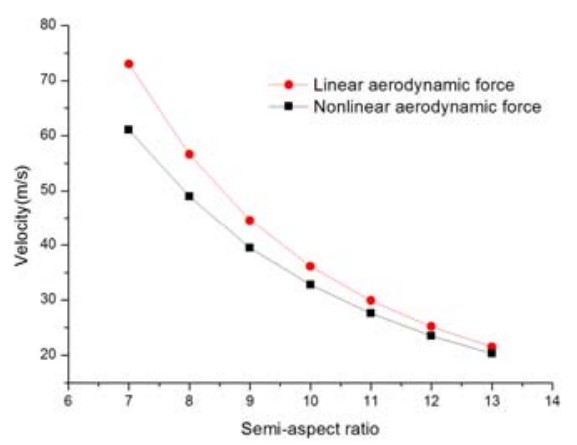

FIGURE III. THE INFLUENCE OF SEMI-ASPECT RATIO ON THE VELOCITY OF THE WING FLUTTER

As shown in Figure 2, the abscissa indicates the mass of nuit length and the ordinate indicates the flutter speed of the wing. It can be seen from the figure that as the density of the wing increases, the flutter speed of the wing also gradually decreases. Meanwhile, the linear aerodynamic forces and the nonlinear aerodynamic forces are also closer to each other, that is, the error of the calculated results by the two methods is smaller. Aspect ratio changes will have a greater impact on the flutter characteristics of the wing, as shown in Figure 3, the abscissa represents the half-aspect ratio of the wing, the vertical axis represents the flutter speed of the wing, As the semi-aspect ratio increases, the flutter speed of the wing drops sharply.

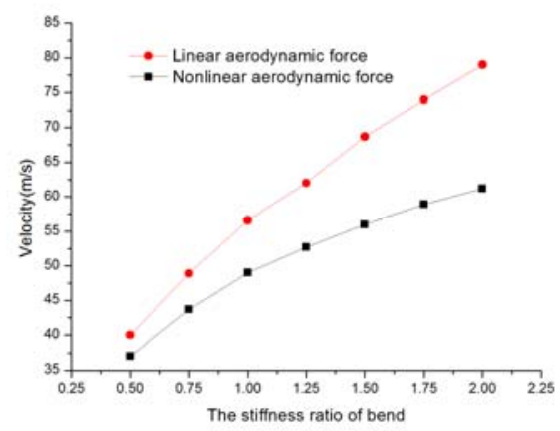

FIGURE IV. THE INFLUENCE OF THE STIFFNESS RATIO OF BEND ON THE VELOCITY OF THE WING FLUTTER 


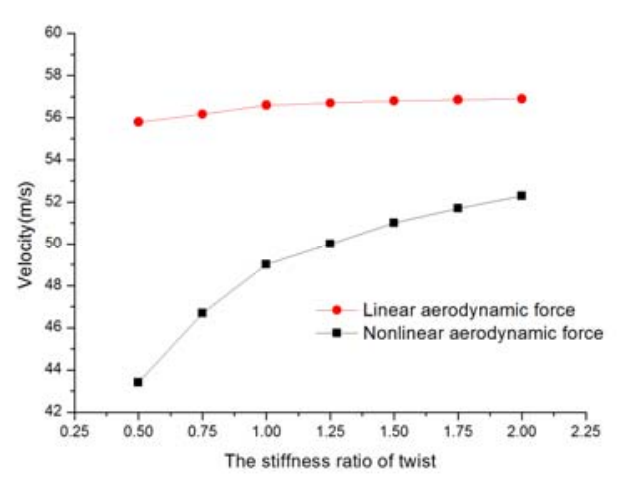

FIGURE V. THE INFLUENCE OF THE STIFFNESS RATIO OF TWIST ON THE VELOCITY OF THE WING FLUTTER

Figure 4 shows the relationship between bending stiffness and flutter speed of the wing. The abscissa indicates the stiffness ratio of bend and the ordinate indicates the flutter speed of the wing. As the bending stiffness increases, the flutter speed of the wing is also increasing. At the same time, the error of linear aerodynamic forces and non-linear aerodynamic forces also increases. Figure 5 shows the relationship between the torsional stiffness of the wing and the flutter speed of the wing. The abscissa shows the stiffness ratio of twist of the wing and the ordinate shows the flutter speed of the wing. The results obtained with flutter using nonlinear aerodynamic forces vary greatly the stiffness ratio of twist changing, especially when the stiffness ratio of twist is smaller, the error between the two is larger, the flutter velocity calculated using the non-linear aerodynamic model more accurately reflects the real wing state.

\section{SUMMARY}

In this paper, the ONERA nonlinear aerodynamic model is applied to the calculation of the wing flutter, meanwhile, the transfer function method is applied to the flutter characteristics analysis of the high aspect ratio wing. The effect of mass of unit length, semi-aspect ratio, the stiffness ratio of twist and bend torsional stiffness on flutter characteristics leads to the following conclusion:

1) The non-linear aerodynamic force calculation results show that the flutter velocity is lower than that obtained by using linear aerodynamic force, which indicates that the aerodynamic nonlinearity reduces the aerodynamic characteristics of the wing and affects the flutter behaviors of wing.

2) When the relative error is small, the linear aerodynamic model with small computational load can be used to calculate. When the error is large, you need to use non-linear aerodynamic calculations to achieve the more realistic wing flutter behaviors.

\section{REFERENCE}

[1] E. Livne. Future of airplane aeroelasticity. Journal of Aircraft. 2003, 40(6):1066-1092.

[2] MJ Patil, DH Hodges, CES Cesnik. Limit cycle oscillations in high-aspect-ratio Wings. Structures, Structural Dynamics \& Materials Conference, 1999, 15(1):107-132
[3] Wang Wei, Zhou Zhou, Zhu Xiaoping, Wang Rui. Static Aeroelastic Characteristics Analysis of a Very Flexible Solar Powered UAV with Geometrical Nonlinear Effect Considered. Journal of Northwestern Polvtechnical University, 2014, 32(4): 499-505.

[4] Wang Wei, Zhou Zhou, Zhu Xiaoping, Wang Rui. Exploring Aeroelastic Stability of Very Flexible Solar Powered UAV with Geometrically Large Deformation. Journal of Northwestern Polvtechnical University, 2015, 33(1): 1-7.

[5] R Palacios, C Cesnik Static. Nonlinear Aeroelasticity of Flexible Slender Wings in Compressible Flow. Aiaa Journal, 2005.

[6] Z Sotoudeh, DH Hodges, CS Chang. Validation Studies for Aeroelastic Trim and Stability of Highly Flexible Aircraft. Journal of Aircraft, 2010, 47(47):1240-1247.

[7] D Tang, A Grasch, EH Dowell. Gust Response for Flexibly Suspended High-Aspect Ratio Wings. Aiaa Journal, 2010, 48(10):2430-2444.

[8] MJ Patil, DH Hodges, CES Cesnik. Nonlinear Aeroelasticity and Flight Dynamics of High-Altitude Long-Endurance Aircraft. Journal of Aircraft, 2012, 38(1):88-94.

[9] M Smith, M Patil, D Hodges. CFD-based analysis of nonlinear aeroelastic behavior of high-aspect ratio wings. Structures, Structural Dynamics \& Materials Conference, 2013.

[10] Zhao Yong Hun. Aeroelastic mechanics and control. Beijing: Science Press, 2006.

[11] Zhou Jian Ping, Lei Yong Jun. Distributed parameter transfer function method. Beijing: Science Press, 2010. 